ESAIM: PROCEEDINGS, October 2007, Vol. 22, 114-117

Gabriel Caloz \& Monique Dauge, Editors

\title{
MATHEMATICAL MODELS IN MICROMAGNETISM: AN INTRODUCTION
}

\author{
Francois Alouges ${ }^{1}$
}

\begin{abstract}
The modelization of ferromagnetic materials obeys the micromagnetic theory proposed by W. F. Brown [4] for static configurations and the Landau-Lifchitz (or Landau-Lifchitz-Gilbert) equations for the dynamics. In this paper, we briefly present these underlying models. Difficulties including physical relevance, numerical simulations and analytic properties of the equations that one wants to keep at the discrete level are discussed.
\end{abstract}

Résumé. La modélisation de matériaux ferromagnétiques suit la théorie du micromagnétisme proposée par Brown pour les configurations statiques (d'équilibre) et les équations de Landau-Lifchitz (ou Landau-Lifchitz-Gilbert) pour la dynamique. Dans ce papier, nous décrivons rapidement les principaux modèles utilisés. Les difficultés incluant la pertinence physique, les simulations numériques ainsi que le respect au niveau discret des propriétés analytiques du modèle sont précisées.

\section{INTRODUCTION}

Ferromagnetic materials are nowadays in the heart of important and/or innovating technological applications. Magnetic storage (hard disks, magnetic tapes, but also magnetic memories MRAMs) is still of considerable interest but one sees also new applications for instance in mobile phones. The model commonly used by physicists to describe the behavior of such material is called micromagnetism and has been proposed by Brown [4] in the 60 's. Static physical configurations are sought as minimizers of an energy functional while dynamical aspects are taken into account by a PDE called Landau-Lifchitz equations. In both cases the unknown is the magnetization which is a vector-field inside the magnetic domain. For an overview of the physical "state-of-the-art" of this domain, we refer the reader to the excellent book by A. Hubert and R. Schäfer [7].

\section{Micromagnetism}

A ferromagnetic material which occupies a domain $\Omega \subset \mathbb{R}^{3}$ is characterized by the presence of a spontaneous magnetization $m$ which is a vector-field of constant magnitude $M_{s}(T)$ throughout $\Omega$ which only depends on the temperature $T$. After a suitable adimensioning ${ }^{1}$, it is not restrictive to assume

$$
|m(x)|=1 \text { a.e. in } \Omega .
$$

The micromagnetic model consists in associating to $m$ a free energy which usually takes the form

$$
E(m)=A \int_{\Omega}|\nabla m|^{2} d x+K \int_{\Omega} \phi(m) d x-\int_{\Omega} H_{e} \cdot m d x+\frac{1}{2} \int_{\mathbb{R}^{3}}\left|H_{d}(m)\right|^{2} d x .
$$

\footnotetext{
1 Laboratoire de Mathématiques, Bât. 425, Université Paris 11, 91405 Orsay Cedex, France

${ }^{1}$ Of course, $M_{S}(T)$ plays a crucial role, in particular for the values of characteristic lengths which appear naturally in the model $[3,9]$.

(C) EDP Sciences, SMAI 2007
} 
Other terms reflecting for more accurate physics (e.g. magnetostriction) can be added to the energy but these four terms already explain a very wide variety of phenomena [7].

Before explaining these four energy terms let us just note that relevant physical configurations of magnetization are sought as solution to the minimization problem

$$
\min _{m \in H^{1}\left(\Omega, \mathbb{S}^{2}\right)} E(m)
$$

where $\mathbb{S}^{2}$ stands for the unit sphere of $\mathbb{R}^{3}$. The first term of the energy is usually called the exchange term, and $A$ the exchange constant. The second term reflects a possibly anisotropic behavior of the crystal composing the ferromagnetic material. Namely, the function $\phi: \mathbb{S}^{2} \rightarrow \mathbb{R}^{+}$is supposed to vanish in a few usually called "preferred" directions $m_{i}$. Due to this anisotropy term, configurations that are locally parallel to these directions are favoured. Typical anisotropies are the uniaxial anisotropy where $\phi(m)=\left(1-(m \cdot u)^{2}\right)$ with only one preferred direction $u$ (here $|u|=1$ ) and the cubic anisotropy for which $\phi$ vanishes on three mutually orthogonal directions. This term is weighted by the anisotropy constant $K$. The last two terms stand for magnetic effects. The first is the exterior energy which accounts for the possible presence of an exterior magnetic field $H_{e}$ applied to the sample (in which case the magnetization tends to align along $H_{e}$ ). The last term of (2) is the so-called "strayfield" energy, and reflects the energy of the stray-field $H_{d}(m)$ induced by the distribution $m$ through Maxwell equations. Namely, $H_{d}(m)$ is obtained by solving the following equations

$$
\left[\begin{array}{l}
\operatorname{curl} H_{d}(m)=0 \text { in } \mathbb{R}^{3} \\
\operatorname{div}\left(H_{d}(m)+m\right)=0 \text { in } \mathbb{R}^{3} \\
H_{d}(m) \rightarrow|x| \rightarrow \infty
\end{array}\right.
$$

Both equations are understood in the sense of distributions in $\mathbb{R}^{3}$, and $m$ is trivially extended in $\mathbb{R}^{3}$ by 0 outside $\Omega$. Writing the stray field as the gradient of a potential $H_{d}(m)=\nabla \phi_{d}(m)$, the former equation rewrites as

$$
-\Delta \phi_{d}(m)=\operatorname{div}\left(m 1_{\Omega}\right) \text { in } \mathbb{R}^{3} .
$$

As a direct consequence, the stray-field energy vanishes whenever $m$ is divergence-free in distributional sense in the whole space $\mathbb{R}^{3}$, that is to say

$$
\left[\begin{array}{l}
\operatorname{div} m=0 \text { in } \mathbb{R}^{3} \\
m \cdot n=0 \text { on } \partial \Omega
\end{array}\right.
$$

where $n$ stands for the unit outward normal of $\Omega$. In particular solving (6) for bidimensional domains and magnetizations $m=\left(m_{1}, m_{2}\right)$ of unit magnitude leads to write $m=\nabla^{\perp} \psi$ where $\psi$ is constant on $\partial \Omega$, and $\psi$ satisfies the eikonal equation

$$
|\nabla \psi|=1 \text { on } \Omega \text {. }
$$

The explicit construction of the viscosity solution of (7) using the method of characteristics is termed in the physics literature by the van den Berg's construction [10]. The complete justification of van den Berg's construction from the three-dimensional model is not fully understood although it has been thoroughly studied (see [6] for a recent review of this subject among others). This construction is one of the key arguments of [9]. Moreover, the numerical computation of the stray field induced by a magnetization distribution is by no means obvious. It involves a non-local operator which after discretization usually leads to a dense matrix. We refer the reader to [8] where this issue is discussed and a fast and accurate method is proposed.

Existence of solutions to (3) is classical and does not pose any difficulty. Uniqueness of such solutions is likely wrong (due to the constraint (1) the problem is not convex), although the energy can be quadratic, for uniaxial anisotropy for instance. Nonetheless, finding algorithms suitable for the numerical computations of equilibrium configurations solutions of (3) is difficult. In [9] is given one of the possible methods while the reader is refered to [1] for the description of a finite element method on this problem.

One of the main mathematical and physical questions is to understand the variety of minimizers and their characteristics when the parameters which balance the energy terms vary. Indeed, if $A$ and $K$ are small 
compared to 1 then the main part of the energy comes from the magnetic terms and it is expected in that regime that solutions tend to recover a behavior close to the one described by the eikonal equation. Conversely, when $A$ is very big compared to $K$ and 1, then the solutions should not vary in space and such regime is called the macrospin approximation. As we see, there are a lot of possibilities which can be analyzed in terms of $\Gamma$-convergence techniques (see [6] and references therein) since this is the good framework for the convergence of minimization problems.

\section{LANDAU-LIFChitZ EQUATIONS}

As far as the dynamics is concerned, the most commonly used model to describe the time evolution of the magnetization is the Landau-Lifchitz equation

$$
\frac{\partial m}{\partial t}=-m \times H(m)+\alpha(H(m)-(H(m) \cdot m) m) .
$$

Here, $\alpha>0$ is a damping parameter and the total magnetic field $H$ (also called the effective field) is given by

$$
H(m)=-\frac{\partial E}{\partial m}=2 A \Delta m-K \nabla_{m} \phi(m)+H_{d}(m)+H_{e} .
$$

It is easily seen that (8) formally preserves the constraint (1) and that it can (still formally) also be written under the Gilbert form (or Landau-Lifchitz-Gilbert)

$$
\frac{\partial m}{\partial t}-\alpha m \times \frac{\partial m}{\partial t}=-\left(1+\alpha^{2}\right) m \times H(m) .
$$

Moreover, multiplying (8) by $H(m)$ and integrating in time leads to the dissipation of energy

$$
\frac{d E(m(t))}{d t}=-\alpha \int_{\Omega}|H(m)-(H(m) \cdot m) m|^{2},
$$

which reflects the fact that only the second term in the right-hand side of (8) dissipates the energy. The first term, however, makes the magnetization turn around the (local) total field $H(m)$ and is termed as the Larmor precession. Moreover, stationary solution of (8) satisfy (also in view of (10))

$$
H(m) \times m=0 \text { in } \Omega,
$$

which is precisely the Euler-Lagrange equations of the minimization problem (3). Therefore, minimizers of $E(m)$ under the constraint (1) are stationary solutions of (8).

Landau-Lifchitz equations are a nonlinear system of partial differential equations for which existence of solutions - when initial and boundary copnditions are supplied - is far from being obvious. In that direction, the classical references are $[2,11]$ for the existence of global weak solutions to (8) and [5] for the existence of strong solutions locally in time or globally for bidimensional domains and initial data of sufficiently small energy. Counterexamples to the uniqueness of weak solutions are also given in [2] in the case where the energy only contains the exchange term.

The linearization of (8) around a minimizer of $E(m)$ leads to a linear behavior of the magnetization distribution in terms of the applied exterior magnetic field $H_{e}$. This linear behavior ${ }^{2}$ is called the magnetic susceptibility and is the subject studied in $[3,8]$ with the help of numerical simulations.

\footnotetext{
${ }^{2}$ More precisely the matrix $\chi: H_{e} \rightarrow \frac{1}{|\Omega|} \int_{\Omega} m$.
} 


\section{Conclusion}

This paper is an introduction to the topics presented in $[3,8,9]$. We have described the models used for analysis and numerical simulations of ferromagnetic materials. Both static configurations and dynamic evolutions of solutions are adressed and we refer the reader to [9], [8] and [3] for more precise applications respectively for static equilibrium configurations, numerical computations issues and the computation of susceptibility response of 3-dimensional magnetic object.

\section{REFERENCES}

[1] F. Alouges, S. Conti, A. De Simone and Y. Pokern, Energetics and switching of quasi-uniform states in small ferromagnetic particles, Mathematical Modelling and Numerical Analysis Vol. 38, N2 (2004) 235-248.

[2] F. Alouges and A. Soyeur, On global weak solutions for Landau-Lifshitz equations, Nonlinear Analysis, Theory, Methods \& Applications, Vol. 18, N. 11 (1992), p. 1071-1084.

[3] F. Boust, N. Vukadinovic and S. LabBÉ, 3D dynamic micromagnetic simulations of susceptibility spectra in soft ferromagnetic particles, in this volume.

[4] W.F. Brown, Micromagnetics, Interscience Publishers, 1963.

[5] G. Carbou and P. Fabrie, Regular solutions for Landau-Lifschitz equation in $\mathbb{R}^{3}$, Commun. Appl. Anal. 5 (2001), no. 1, $17-30$.

[6] A. DeSimone, R. Kohn, S. Müller and F. Otto, Recent analytical developments in micromagnetics, in The Science of Hysteresis, G. Bertotti and I. Mayergoyz, Eds., vol. 2. Academic Press (2006), ch. 4, pp. 269-381.

[7] A. Hubert and R. Schäfer, Magnetic Domains : The Analysis of Magnetic Microstructures, Springer-Verglag, 2000.

[8] S. LABBÉ, Numerical simulations of ferromagnetic materials, in this volume.

[9] J.-C. Toussaint, O. Fruchart and F. Cheynis, Micromagnetic modeling on self-assembled iron nanostructures, in this volume.

[10] H. A. M. van DEN Berg, Self-consistent domain theory in soft ferromagnetic media. II. Basic domain structures in thin films objects, J. Appl. Phys. 60 (1986), 1104-1113.

[11] A. Visintin, On Landau-Lifshitz' equations for ferromagnetism, Japan J. Appl. Math. 2, no. 1 (1985), 69-84. 